\title{
A Full Time-Domain Approach to Spatio-Temporal Dynamics of Active Semiconductor Devices
}

\author{
Klaus Boehringer and Ortwin Hess \\ Advanced Technology Institute, University of Surrey, Guildford, Surrey, GU2 7XH, United Kingdom
}

\begin{abstract}
We present a finite-difference full time-domain model including the macroscopic Maxwell curl equations and the band-resolved semiconductor Bloch equations. A key element of our novel formulation is that neither the slowly varying amplitude nor the rotating wave approximation are necessary. This makes the model accurate to a broad frequency range. The propagation, amplification and reshaping of femtosecond pulses in active semiconductor amplifiers as well as optically pumped monolithic disk lasers with sub-wavelength refractive index structures and nonlinear gain or absorber elements are simulated.
\end{abstract}

\section{INTRODUCTION}

With the size of optical devices being moved more and more to the length scale of the wavelength or even less, the laser cavities feature spatially strong localised cold-cavity modes. Also, crucial ideas behind new laser designs capitalise on the broad spectral bandwidth of the semiconductor gain media and exploit the nonlinear multi-mode dynamics. Therefore, a realistic theoretical model should address the sub-wavelength length scales together with multi-frequency behaviour. The usual approach of expanding the fields in terms of counterpropagating plane waves, a partial transformation to Fourier (frequency and momentum) space, and the application of the paraxial, slowly varying amplitude and rotating wave approximations obviously fail in such situations. Here we present a model which solves the full Maxwell equations combined with a realistic semiconductor gain description accurately over a broad frequency range. In the next section we give an outline of the semiconductor Maxwell Bloch equations in full timedomain.

\section{BASIC EQUATIONS}

Besides the description of the gain media a semiclassical laser model must include equations specifying the dynamics of the optical fields. For a linearly transverse-polarised wave ( $\left.H=H_{x}(z, t), E=E_{y}(z, t)\right)$ propagating in $z$-direction the Ampère's and Faraday's law have the form $\partial_{z} H-\partial_{t} D=0$, $\partial_{z} E-\mu_{0} \partial_{t} H=0$. Generally, the interface to the semiconductor gain material response is given by the macroscopic induced polarisation $D=\epsilon_{0} \epsilon(z) E+\Gamma_{x, y} N_{\mathrm{QW}} / L_{\mathrm{ref}} \cdot P$ which is the spectral summation over the microscopic, band-resolved quantity. The semiconductor Bloch equations provide a realistic gain description [1]. Here we have transformed the wellknown set of equations derived from density matrix theory [2] (with additional phenomenological terms) to the following

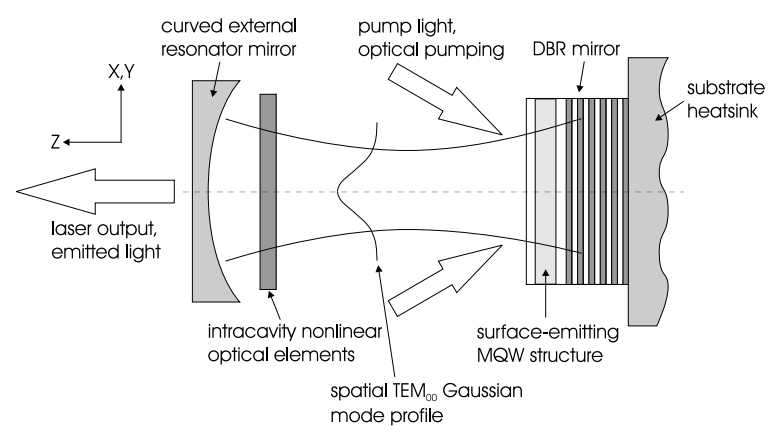

Fig. 1. Schematic structure of a optically pumped VECSEL: Starting from the three-dimensional full-vectorial Maxwell curl equations we concentrate on the longitudinal propagation direction and assume constant profiles in the perpendicular directions. The spatio-temporal dynamics of the electromagnetic fields can thus be qualified by the Maxwell curl equations in one dimension in an effective way, the other directions can be included via confinement factors $\Gamma_{x, y}$ (the overlap integrals of the tranverse mode profiles with the active media) und by loss terms.

equivalent formulation for the microscopic polarisation

$$
\begin{aligned}
& \partial_{t}^{2} \mathbf{p}_{\mathbf{k}}+2 \gamma_{\mathbf{k}}^{p} \partial_{t} \mathbf{p}_{\mathbf{k}}+\left(\Omega_{\mathbf{k}}^{2}+\left(\gamma_{\mathbf{k}}^{p}\right)^{2}\right) \mathrm{p}_{\mathbf{k}}= \\
& -\Omega_{\mathbf{k}} \frac{M^{e h} E}{\hbar}\left(n_{\mathbf{k}}^{e}+n_{-\mathbf{k}}^{h}-1\right), \hbar \Omega_{\mathbf{k}}=E_{\text {gap }}+E_{\mathbf{k}}^{e}+E_{-\mathbf{k}}^{h}
\end{aligned}
$$

and the non-equilibrium carrier distributions

$$
\begin{gathered}
\partial_{t} n_{\mathbf{k}}^{e}=2 \frac{M^{e h} E}{\hbar} \frac{1}{\Omega_{\mathbf{k}}}\left(\gamma_{\mathbf{k}}^{p} \mathrm{p}_{\mathbf{k}}+\partial_{t} \mathbf{p}_{\mathbf{k}}\right)-\gamma_{\mathbf{k}}^{e}\left(n_{\mathbf{k}}^{e}-f_{\mathbf{k}}^{e}\right) \\
+\Lambda \frac{f_{\mathbf{k}}^{e}\left(1-n_{\mathbf{k}}^{e}\right)}{\sum_{\mathbf{k}} f_{\mathbf{k}}^{e}\left(1-n_{\mathbf{k}}^{e}\right)}-\gamma^{\mathrm{nr}} n_{\mathbf{k}}^{e}-\gamma_{\mathbf{k}}^{\mathrm{sp}} n_{\mathbf{k}}^{e} n_{-\mathbf{k}}^{h}-\gamma_{\mathbf{k}}^{\text {Auger }}
\end{gathered}
$$

\section{NUMERICAL IMPLEMENTATION}

The numerical implementation of the semiconductor Maxwell Bloch equations within the finite-difference timedomain (FDTD) framework builds on an adequate choice of discretisation schemes and the correct centring of fields in time and space. A central finite-difference approximation of the derivatives of the fields is applied, and the fundamental idea of the Yee discretisation scheme to arrange the electric and magnetic field quantities on staggered grids, on temporal and spatial grids with relative offsets of half steps is taken on. At the boundary of our finite analysis window boundary conditions according to Mur are applied, well defined external optical fields are injected into the system using the totalfield/scattered-field (TFSF) technique [3]. $n_{\mathbf{k}}^{e}, p_{\mathbf{k}}$ are fixed to the same grid positions with the electric field quantities. The crucial property of our discretisation and integration scheme 


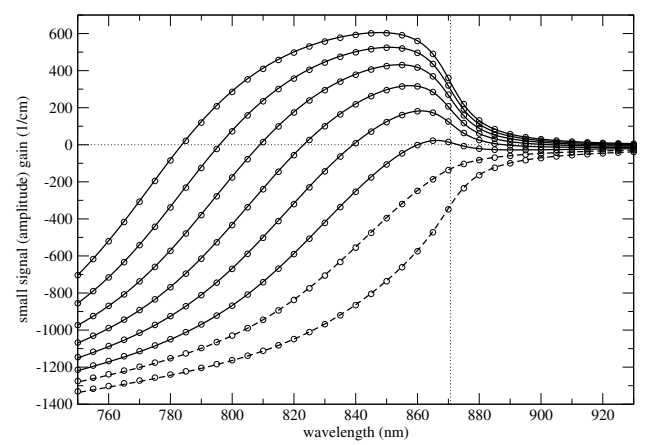

Fig. 2. Small signal amplitude gain vs. optical wavelength for various sheet carrier densities $\left\{0.5 \ldots 4 \cdot 10^{12} \mathrm{~cm}^{-2}\right\}$. One can see the transition from an absorber to a gain medium, the transparency density is $\approx 1.5 \cdot 10^{12} \mathrm{~cm}^{-2}$. The very close agreement between the results obtained from standard free-carrier theory [1] (circles) and that calculated from the full time-domain simulations (lines) is a verification for our novel approach.

is the fact that the combined active material and passive refractive index structure model in full time-domain can be implemented in a fully explicit and therefore computationally efficient manner.

\section{Simulation Results}

\section{A. Semiconductor Optical Amplifiers}

As a first test system we probe a semiconductor amplifier structure (with GaAs quantum wells) with a weak femtosecond pulse to scan the non-instantaneous polarisation response of the gain medium. The time series are recorded at a point $z_{L}$ for the active structure for different carrier sheet densities and for comparison also for the passive structure. From the time series and the subsequent Fourier transforms we calculate the small signal amplitude gain spectra $\partial_{z} E=g(N, \lambda) E$ according to $g=1 / L_{\text {act }} \cdot \ln \left(E_{\text {act }}\left(z_{L}, N, \lambda\right) / E_{\text {pas }}\left(z_{L}, \lambda\right)\right.$ ) (see Fig. 2). The interaction of the optical fields with the active gain material is for increased field amplitudes (in the nonlinear regime) rather complex since we can no longer assume that the test pulse is just sampling the optoelectronic properties of the semiconductor gain material. It drastically changes the state of the active gain medium. The spectral and over-all depletion of carriers (spectral and spatial hole burning) reduces the amplification of the pulse (gain saturation) [4], this behaviour is properly included in our full time-domain model.

\section{B. Optically Pumped Disk Lasers}

The concept of the novel laser structure VECSEL (verticalexternal-cavity surface-emitting laser) was at first suggested in [5]. Thereby one Bragg mirror stack of a VCSEL is replaced by an external resonator. The structure is optically pumped (thus no need for doping), the aim is to combine the neardiffraction-limited beam quality of a VCSEL with the scalability to a high output power laser device in continuous wave operation [6]. Moreover, the possibility to place additional elements into the external cavity configuration, like saturable absorber mirror elements (SESAM) for passive mode-locking [7], is very attractive (see Fig. 1). Fig. 3 demonstrate the strengths of our method.
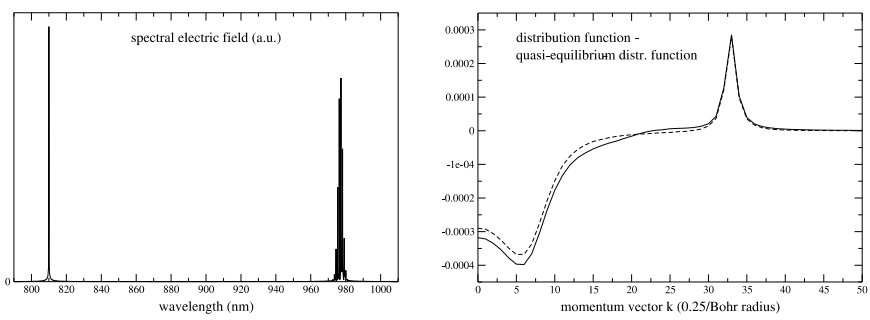

Fig. 3. Left: Spectrum vs. wavelength of an optically pumped multi-mode semiconductor laser. One can clearly see the optical pump at $\approx 808 \mathrm{~nm}$ and several longitudinal cavity modes at around $980 \mathrm{~nm}$ with a spectral spacing of $\approx 1 \mathrm{~nm}$ (length of the external resonator $500 \mu \mathrm{m}$ ). It is impossible to model this optical pumping process on the basis of a homogeneously broadened two-level system or with a model using the SVAA or RWA (This is due to the fact that with these approximations the optical fields are not accurate for the lasing frequencies and for the pumping frequency at the same time, one has to concentrate on one of the processes, like the pumping and subsequent relaxation.). Right: The spectral hole burning and the spectral selective carrier generation (optical pumping) in the distribution functions of the electrons $\left(\Delta n_{\mathbf{k}}^{e}=n_{\mathbf{k}}^{e}-f_{\mathbf{k}}^{e}\right)$ and holes $\left(\Delta n_{-\mathbf{k}}^{h}\right.$, dashed line $)$.

\section{CONClusion}

In this paper we have presented the semiconductor Maxwell Bloch equations in full time-domain, a novel approach to simulate active semiconductor optical devices. Because of the fully explicit discretisation scheme and numerical implementation the algorithm is very efficient, yet absolutely accurate (over a broad spectral range) as shown in the calculations of the small signal gain spectra. First numerical results of the time dynamics of active laser devices indicate the potential of our model. In the future we will address the propagation, amplification and reshaping of few-cycle optical pulses in semiconductor structures. The recovery dynamics of fast saturable absorber elements and the mechanisms behind the generation and shaping of femtosecond pulses by passive mode-locking are other foci.

\section{ACKNOWLEDGMENT}

The authors would like to thank Andreas Klaedtke and Joachim Hamm for stimulating discussions and for the assistance in the numerical implementation of the model.

\section{REFERENCES}

[1] W. W. Chow and S. W. Koch, Semiconductor-Laser Fundamentals: Physics of the Gain Materials. Springer-Verlag, 1999.

[2] O. Hess and T. Kuhn, "Maxwell-Bloch equations for spatially inhomogeneous semiconductor lasers. I. Theoretical formulation," Phys. Rev. A, vol. 54, no. 4, pp. 3347-3359, Oct. 1996.

[3] A. Taflove and S. C. Hagness, Computational Electrodynamics: The Finite-Difference Time-Domain Method, 2nd ed. Artech House, 2000.

[4] P. P. Vasil'ev, I. H. White, and J. Gowar, "Fast phenomena in semiconductor lasers," Rep. Prog. Phys., vol. 63, no. 12, pp. 1997-2042, Dec. 2000.

[5] M. Kuznetsov, F. Hakimi, R. Sprague, and A. Mooradian, "Design and characteristics of high-power $(>0.5-\mathrm{W} \mathrm{CW})$ diode-pumped vertical-external-cavity surface-emitting semiconductor lasers with circular TEM 00 beams," IEEE J. Select. Topics Quantum Electron., vol. 5, no. 3, pp. 561-573, 1999.

[6] A. C. Tropper, H. D. Foreman, A. Garnache, K. G. Wilcox, and S. H. Hoogland, "Vertical-external-cavity semiconductor lasers," J. Phys. D: Appl. Phys., vol. 37, pp. R75-R85, Apr. 2004.

[7] U. Keller, "Recent developments in compact ultrafast lasers," Nature, vol. 424 , pp. 831-838, Aug. 2003. 